\title{
Perspectives interdisciplinaires sur le travail et la santé
}

9-2 | 2007

Mutations du travail face aux défis technologiques et à leurs incidences sur le travail

\section{Introduction}

Marc-Éric Bobillier Chaumon et Michel Dubois

\section{(2) OpenEdition \\ Journals}

Édition électronique

URL : http://journals.openedition.org/pistes/2958

DOI : 10.4000/pistes.2958

ISSN : 1481-9384

Éditeur

Les Amis de PISTES

Référence électronique

Marc-Éric Bobillier Chaumon et Michel Dubois, «Introduction », Perspectives interdisciplinaires sur le travail et la santé [En ligne], 9-2 | 2007, mis en ligne le 01 octobre 2007, consulté le 01 mai 2019. URL : http://journals.openedition.org/pistes/2958; DOI : 10.4000/pistes.2958

Ce document a été généré automatiquement le 1 mai 2019.

\section{(c) (i) ()}

Pistes est mis à disposition selon les termes de la licence Creative Commons Attribution - Pas d'Utilisation Commerciale - Pas de Modification 4.0 International. 


\title{
Introduction
}

\author{
Marc-Éric Bobillier Chaumon et Michel Dubois
}

1 L'importance des technologies d'information demeure toujours d'actualité, car elle concerne directement la gestion des activités de travail par les professionnels, les liens avec l'évolution des conditions de travail et les conséquences sur la santé et le bien-être des salariés. De l'informatique des années 1960 aux technologies de l'information et de la communication (TIC), fruits de la convergence numérique : où en est-on de la diffusion des TIC dans les entreprises et dans les administrations et quels sont les principaux enjeux actuels?

2 La société de l'information est en fait le résultat de diverses interactions entre les innovations technologiques et toutes les autres innovations qui ont profondément modifié la société et les organisations : innovations commerciales (individualisation, réduction des délais, recherche de la flexibilité commerciale...), innovations organisationnelles (gestion des temps, des flux, des projets, recherche de la flexibilité organisationnelle...) et innovations sociales (réduction du temps de travail, intensification, individualisation, précarisation, recherche de la flexibilité sociale...). Les dimensions $\mathrm{du}$ travail ont aussi fortement évolué: abstraction, interactivité, chronophagie, vulnérabilité, nomadisme, télétravail, transparence... L'évolution des TIC nécessite, hors de la vision d'un simple déterminisme technologique, de s'intéresser aux diverses transformations du travail à prendre en compte en fonction des capacités des utilisateurs. Parallèlement aux apports certains des TIC dans les activités de travail, plusieurs autres aspects sont à considérer en lien plus ou moins direct avec la santé des travailleurs : émergence de nouvelles compétences, surcharge d'information, suractivité, modification du rapport au client, conflits de buts, conditions du travail en réseau, gestion du handicap, etc.

3 Les articles présentés ont pour vocation, chacun à leur manière, de rendre compte de cette complexité d'approche. Ils sont, pour la plupart, issus de communications présentées à l'École Centrale de Lyon (E.C.L.), lors d'une journée d'étude thématique ${ }^{1}$ intitulée "Les mutations du travail face aux défis technologiques: questionnements psychologiques, sociologiques et éthiques». Certaines communications ont été retravaillées 
par les auteurs par suite de la demande des éditeurs de ce numéro spécial. D’autres auteurs nous ont aussi fait l'honneur d'enrichir les communications initiales de la journée thématique par d'autres contributions. Plusieurs types de questions sont abordés.

4 Un premier ensemble de travaux traite plus particulièrement des relations entre "TIC, usages, organisation et santé ». Robert Picard s'intéresse aux nombreux changements organisationnels associés à (ou provoqués par) l'introduction d'outils de technologies de l'information ou de communication (TIC). La taille des systèmes d'information mis en place (ERP), leur caractère exogène par rapport aux pratiques antérieures de l'entreprise posent des défis relativement nouveaux, et pas toujours faciles à prendre en charge pour les acteurs de l'organisation. L'auteur montre, à travers deux situations concrètes, observées dans un grand groupe de service, comment les métiers des utilisateurs, mais aussi ceux des informaticiens eux-mêmes, sont profondément affectés par l'introduction des TIC. Éric Brangier et Sonia Hammès présentent un modèle d'approche, à la fois conceptuel et méthodologique, pour aborder la question de la relation entre l'homme, la technologie et l'organisation en insistant sur la notion de "symbiose ». Après un rappel des travaux initiaux sur le développement des technologies, les auteurs insistent sur les processus qui favorisent cette symbiose (recherche de fonctionnalités adaptées, définition d'une utilisabilité optimisée, régulation des processus socio-organisationnels). La prise en compte de ces processus permet d'adopter, pour les auteurs, une démarche prospective et méthodologique pour la conception de nouvelles technologies. Cet article propose donc une alternative aux modèles qui insistent sur les conditions d'acceptation des technologies par l'humain en montrant qu'aujourd'hui les technologies contemporaines sont en passe de devenir des «symbiotes» qui, d'un point de vue métaphorique, se couplent avec les individus.

Un second corps de travaux met plus l'accent sur les liens entre «TIC, intensification du travail et santé ». Jacqueline Vacherand-Revel montre l'évolution croissante de nombreuses organisations vers des configurations structurelles plus flexibles, et le déploiement de leurs activités collaboratives sur plusieurs sites distants. Ces changements s'accompagnent d'une mutation des pratiques professionnelles dans de nouveaux environnements technologiques souvent très sophistiqués et en constante évolution. Ce type de médiatisation permet d'assister la coopération en offrant des modalités d'interaction en temps réel (synchrone) et des espaces virtuels de travail communs. Ainsi, des collectifs : équipes de projet ou groupes plus larges, constitués à l'échelle nationale ou internationale, avec des modes de fonctionnement déjà éprouvés ou en cours d'élaboration, dans des configurations de travail intra ou interentreprises, sont confrontés à l'intégration de ces technologies dans leur activité. Ils doivent définir de nouvelles pratiques collectives médiatisées qui comportent une régularité et une densité des échanges ainsi qu'une forte interdépendance entre les acteurs pour mener à bien des tâches communes, intellectuellement et techniquement élaborées. Dans ces contextes, les collectifs sont dits distribués pour désigner à la fois la distance géographique entre les différents sites de travail, les contraintes spatio-temporelles auxquelles ils sont confrontés, mais également souligner la distance en matière de ressources, d'expériences ou de perspectives hétérogènes des acteurs. En regard des enjeux socio-économiques qui président à l'introduction de ces nouvelles pratiques collectives distribuées, force est de constater, une nouvelle fois, le décalage entre les potentialités techniques et leur mise en œuvre sociale et organisationnelle. Actuellement, les expériences significatives sont encore largement expérimentales ou réservées à des milieux singulièrement limités. Sur 
le plan scientifique, comprendre ces phénomènes conduit à interroger la nature des interactions et des jeux sociaux qui orchestrent le travail coopératif dans sa relation aux artefacts et oblige à une prise de conscience nouvelle de ce qu'implique une réelle coopération et de ses multiples déterminants. Le texte de l'auteur est illustré par différentes recherches qui interrogent sous un angle inédit ces nouvelles pratiques de coopération au sein de collectifs distribués engagés dans des activités de co-conception sur plusieurs sites distants. Michel Dubois et Marc Éric Bobillier-Chaumon s'intéressent aux effets des technologies d'information (E.services: E.banking ou E.administration) dédiées aux usagers dans les relations de services ultérieures en face à face avec les agents. À travers deux études empiriques (secteur bancaire et secteur administratif), les résultats montrent que les expertises acquises par les usagers via les E.services ont un certain nombre de répercussions sur l'activité des professionnels: développement de compétences, autonomie, implication, mais aussi un ensemble d'engagements pour réguler les différentes modalités d'intensification de la charge de travail (apprentissage, comportements d'adaptation) ainsi que des impacts sur la santé (stress). La dématérialisation et l'automatisation des traitements administratifs, la virtualisation des relations avec le bénéficiaire, la réduction des marges de manœuvre, ou encore la recomposition des réseaux et des rapports au travail... sont quelques-unes des grandes évolutions observées par suite des développements des E.services.

6 Enfin, un troisième corps de recherches fait ressortir la dimension paradoxale des TIC entre risque d'exclusion et environnement "capacitant». Pierre Pavageau, Adelaïde Nascimento et Pierre Falzon insistent sur les risques d'exclusion via les TIC dans un contexte de transformation organisationnelle. Leur recherche réalisée au sein de deux structures repose sur une approche triaxiale de la santé et sur la notion d'environnement capacitant. Les différentes analyses montrent que contrairement aux idées reçues, l'âge n'apparaît pas comme un facteur prédominant d'exclusion et que la mise en place de nouvelles technologies est plutôt vécue comme positive. Au-delà du changement, les auteurs mettent l'accent sur la vigilance toute particulière à accorder aux différents processus d'adaptation. Jean-Claude Sperandio fait ressortir la dimension paradoxale des TIC qui peuvent, pour les personnes handicapées ou âgées, être soit des obstacles redoutables, soit au contraire apporter des solutions efficaces aux problèmes que rencontrent ces personnes. Pour l'auteur, tout dépend en fait des applications que l'on en fait. Il insiste sur la nécessité, lors de la conception initiale de divers objets ordinaires de la vie courante, mais aussi des aides techniques spécialisées, du logement, des transports publics, des postes de travail, etc., de tenir compte, dès l'amont, de l'existence de personnes handicapées ou très âgées dans la population générale. Il souligne aussi, à travers de nombreux exemples, des difficultés méthodologiques relatives à l'analyse des activités et à l'évaluation d'objets techniques pour les personnes handicapées ou âgées.

7 Enfin, pour conclure, Sandrine Caroly tente, à partir de ces différentes contributions, d'appréhender quelles sont les mutations du travail face aux défis technologiques et essaie de mettre en perspective leurs incidences en matière de santé au travail.

8 Les différentes contributions de ce numéro spécial offrent aux praticiens, aux étudiants et enseignants chercheurs un aperçu de différentes approches possibles des évolutions du travail face aux défis technologiques. Elles montrent que bien souvent la société de l'information a des effets contradictoires sur les conditions de travail et de vie et affirment la nécessité d'élargir le plus possible l'approche de l'articulation TIC et travail en la dotant d'outils conceptuels et méthodologiques rigoureux. L'ensemble de ces 
productions fait ressortir qu'il est impossible de totalement circonscrire les relations complexes et multidimensionnelles entre technologie, travail et santé. L'apport de l'ergonomie et de la psychologie du travail, mais aussi de toutes les disciplines de sciences humaines et sociales, peut être justement de faire reconnaître l'importance de ces évolutions et de faire comprendre la complexité des interactions qui en découlent pour améliorer l'efficacité des actions de correction et de prévention des risques réels et/ou potentiels. Nous espérons que le lecteur trouvera dans ce numéro spécial, dense, ouvert et bien documenté, des éléments d'information et de réflexion lui permettant d'envisager sous des angles pluriels et complémentaires l'approche des TIC dans le travail.

\section{NOTES}

1. Cette journée était organisée par les membres fondateurs du Groupe de Recherche en Ergonomie et Psychologie du Travail (GREPSYT) qui est un réseau de collaboration entre ergonomes et psychologues du travail de la région Rhône-Alpes issus de l'Université de Grenoble (Université Pierre Mendès France), de l'Université de Lyon (Université Lumière Lyon II), de l'Institut d'études du Travail de Lyon et de l'École Centrale de Lyon. Cette journée était soutenue par les Conseils scientifiques de l'UPMF de Grenoble et de l'Université Lumière de Lyon 2. 